Isidore Fleischer, Centre de Recherches Mathématiques, Université de Montréal, C.P. 6128-A, Montréal, Quebec H3C 3J7, Canada e-mail: fleischi@@CRM.UMontreal.CA

\title{
THE CONVERGENCE CONTENT OF THE INTEGRAL CONVERGENCE THEOREM
}

Recently Bartle [B], modifying a convergence theorem of Gordon, has established necessary and sufficient conditions for a Henstock integral convergence theorem. We point out that this result is not specific to the Henstock, or indeed to any definition of, an integral - rather, it is a result about an arbitrary limit process and can thus be formulated in the setting of abstract convergence theory.

The convergence structure will be treated by means of nets. These are maps from directed sets, here to the reals. A set becomes directed by a transitive relation $>$ such that every finite subset has a common greater element. If the directed set is the naturals with their total order, one gets a real sequence; if it is the finite tagged partitions of an interval into non-overlapping subintervals, ordered opposite to max $\left|x_{i}-x_{i-1}\right|$ (the "greater" sum has smaller maximum subinterval length), one has a Riemann integral net $\sum f\left(t_{i}\right)\left(x_{i}-x_{i-1}\right)$; for the Henstock integral one could take pairs consisting of a $\delta$-fine tagged partition and positive function $\delta$, ordered opposite to the pointwise order on the $\delta$.

The usual notions for sequences: convergence, Cauchy, lim sup, $\ldots$ are defined for nets just as for sequences - use the sequential definitions construing the indices as drawn from the directed set.

An integral convergence theorem deals with a sequence of integrable functions $f_{k}$, thus for which the integration net $\sum f_{k}\left(t_{i}\right)\left(x_{i}-x_{i-1}\right)$ converges for each $k$ to a limit $\int f_{k}$, and investigates convergence in $k$ of $\int f_{k}$ and identification of its limit. This sum, as function of $k$ and $P$, will henceforth be replaced by an abstract doubly indexed net $S(k, P)$, for which $k$ and $P$ run through arbitrary directed sets rather than the naturals and tagged partitions. It is assumed that one has the limit for fixed $k, \lim _{P} S(k, P)$, whose limit in $k$ is

\footnotetext{
Mathematical Reviews subject classification: Primary: 26A39 Secondary: 20A03, 28A20 $40 \mathrm{~A} 10$
}

Received by the editors July 7, 1995 
sought. This is the "repeated limit" [G] $\lim _{k} \lim _{P} S(k, P)$, whose existence entails that of the "generalized repeated limit" or, as I prefer to call it, the iterated limit

$$
\lim _{k} \varlimsup_{P} S(k, P)=\lim _{k}\left[\underline{\lim }_{P} S(k, P), \varlimsup_{P} S(k, P)\right]
$$

interpreted as the common limit of the end points of the interval bounded by the $\liminf \operatorname{ind}_{P}$ and $\lim \sup _{P}$.

Three ingredients will make up the proof of the net convergence theorem (stated at the end):

If $S(k, P)$ converges for every $k$, then the repeated limit exists if and only if the iterated limit exists and the two are then equal.

By the way, this is the content of Henstock's necessary and sufficient Theorem 11.2 on p. 107 of $[\mathrm{H}]$.

Next, convergence to the iterated limit may be formulated as the Cauchy condition:

$$
\lim _{k, j} \varlimsup_{P, Q}[S(k, P)-S(j, Q)]=0
$$

or, spelled out (and using the directedness of the index sets): For every $\varepsilon>0$ there exists a $k(\varepsilon)$ such that for every $k, j>k(\varepsilon)$ there is a $P(k, j)$ such that for $P, Q>P(k, j),|S(k, P)-S(j, Q)|<\varepsilon$. If in addition $S(k, P)$ is known to converge in $P$ for every $k$, then one can choose the $P(k, j)$ so that $S(k, P)$ is close to $S\left(k, P^{\prime}\right), S(j, Q)$ is close to $S\left(j, Q^{\prime}\right)$ for $P, P^{\prime}, Q, Q^{\prime}>P(k, j)$ and so obtain the Cauchy condition from the more special

$$
\lim _{k, j} \varlimsup_{P}[S(k, P)-S(j, Q)]=0
$$

Finally, to have another net, $T(P)$, also indexed by the $P$, converge to this iterated limit comes to $T(P)$ close to $S(k, P)$ for sufficiently large $P$ and arbitrarily large $k, Q(k)$ - hence, by convergence to the iterated limit, for sufficiently large $k, Q(k)$. This hypothesis can be combined with the Cauchy requirement, as

$$
\lim _{k} \varlimsup_{P}[S(k, P)-T(P)]=0 .
$$

In the presence of convergence of $S(k, P)$ in $P$ for every $k$, this is thus a necessary and sufficient condition for the existence of the repeated limit, that of the $T(P)$, and their equality. 
The Integral Convergence Theorem

\section{References}

[B] R. G. Bartle, A convergence theorem for generalized Riemann integrals, Real Anal. Exchange, 20 (1995), 119-124.

[G] L. M. Graves, The Theory of Functions of Real Variables, McGraw-Hill, New York, 1956.

[H] R. Henstock, Lectures on the Theory of Integration, World Scientific, Singapore, 1988. 